\title{
Fire Model Validation for Gas Temperatures and Radiative/Convective Partitioned Heat Flux
}

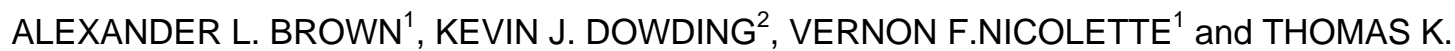 \\ BLANCHAT ${ }^{1}$ \\ ${ }^{1}$ Fire \& Aerosol Science Department \\ ${ }^{2}$ Validation and Uncertainty Quantification Processes \\ Sandia National Laboratories (SNL) \\ PO Box 5800 \\ Albuquerque, NM 87185, USA
}

\begin{abstract}
A recent series of fire experiments has provided previously unavailable high-fidelity data for heat fluxes and gas temperature in a large-scale fire. These include histograms of temporally-resolved gas temperature measurements, which are helpful in assessing the time accuracy of a transient code. A convergence analysis has been performed to determine the appropriate code requirements for comparing with these new data. The convergence analysis suggests a significant benefit to employing higher resolution than was readily possible for these comparisons. Comparisons are made to the data for measured values including partitioned convective/radiative heat flux, and gas phase temperatures. Uncertainty analyses show that the uncertainties due to grid refinement are on the order of those from model input parameter uncertainties. The gas phase temperature results suggest the need for increased mesh resolution to resolve the temperature distributions.
\end{abstract}

KEYWORDS: CFD, modeling, validation, transportation fires

\section{INTRODUCTION}

Modern scientific and engineering methods have provided advancements in experimental and simulation capabilities involving liquid fuel fires which are often present in transportation accidents. Studying such fire scenarios presents certain challenges. The environment is adverse, and extracting confident data from a fire is challenged by the extreme temperatures and fouling from combustion reactants, intermediates, and products. The physics of the fire are complex. Among others, chemistry, turbulence, radiation, diffusion, and soot present challenges to the modeling of a fire. And the range of length and time scales involved in practical sized fires often creates further difficulties. There are consequences to these challenges. One is that there are often insufficient data to fully understand the complexities of even some simplified laboratory test fires. Also, much of the data are poorly resolved temporally. Thermocouples have response times on the order of seconds, as do most instruments sampling data in a fire. This means that most data available are only appropriate for comparing time average values. A further complexity is that modeling and simulation tools are often over-simplified or are missing necessary physical model descriptions for accurate modeling of a fire.

When employing a model for engineering analysis, it is known that the model approximates the real physical processes. Validation is a technique used to establish the accuracy with which a model captures the effects of a physical process. Validation is limited by the data available, and often the simulation produces outputs that cannot be measured and hence validated. Although simulations are deterministic, we recognize that the effect of modeling assumptions can be studied through sensitivity and uncertainty analyses.

High-fidelity datasets have recently become available that were designed to provide unique data for validation. These tests were performed with methanol fuel to reduce soot formation for the optical diagnostics, and are documented in [1-3]. The tests were performed in the new Fire Laboratory for the Accreditation of Models and Experiments (FLAME) facility at Sandia National Laboratories, which was designed with strict control of boundary conditions for collecting validation-quality data. Besides the well characterized boundary conditions, new diagnostics have been fielded, which include detailed data on the heat transfer to an object in the fire and Coherent Anti-Stokes Raman Spectroscopy (CARS) for highresolution temperature and species [2]. The CARS technique has sufficient spatial and temporal resolution 
(order of $1 \mathrm{~cm}$ and $10 \mathrm{~ns}$ ) to validate the time resolved calculations that can be performed with a Large Eddy Simulation (LES) or similar type of CFD code. This work represents the first known comparison (to the authors' knowledge) of a CFD fire code to spatially and temporally resolved (CARS) temperature measurements for a fire of this scale.

The present study contributes to the validation of the fire code Fuego [4]. While this is of significant interest to users of the code, it is of broader interest to the fire science community because the study suggests resolution requirements relevant for similar fire codes that attempt to simulate this class of problems. Additionally, procedures to address model uncertainty due to mesh resolution and input parameter uncertainty are applied. Addressing these uncertainties is an important aspect of model validation and is demonstrated in this paper.

\section{METHODS}

The simulation tool used in these comparisons is Fuego, and has been developed at Sandia National Laboratories for fire simulations as part of the US Department of Energy's Advanced Simulation and Computing (ASC) program (see for example [5]). The code is designed to scale for parallel calculations so that very high resolution problems can be addressed.

To model these methanol fires, the Temporally Filtered Navier Stokes (TFNS) turbulence model is used as described in [6]. The TFNS model is similar to the more common LES codes, except a temporal filter is employed instead of a spatial filter, much like Girimaji's PANS model [7]. Thermodynamics properties for methanol, combustion products, and air are taken from a standard thermodynamics library. Since methanol fires produce little to no soot, the Tesner-based [8] soot model was deactivated. The reactions were modeled as mixing limited, employing the assumptions of the Magnussen Eddy Dissipation concept (EDC) model [9]. Radiation was modeled with the Discrete Ordinates (DO) model with a quadrature of 4. A gray gas approximation was used, with $\mathrm{CO}_{2}$ and $\mathrm{H}_{2} \mathrm{O}$ gas radiation properties taken from Leckner [10]. The spectral characteristics of the methanol fires are significant, and the gray approximation is recognized as an assumption with uncertainty.

Comparisons are made to data that are described in [1-3]. These tests were performed at the FLAME facility at Sandia National Laboratories in Albuquerque, NM. The facility and set-up are also illustrated in Fig.1. The FLAME facility is an $18.4 \mathrm{~m}$ diameter, $12.2 \mathrm{~m}$ high cylindrical test cell (from ground to the lowest section of the ceiling). After an $18^{\circ}$ conical transition in the ceiling, the air exhausts to a $4.9 \mathrm{~m}$ diameter duct (included in the model) that transitions again to a smaller rectangular duct (omitted from the model). Air enters at low velocity in the floor of the basement through a $60 \mathrm{~m}^{2}$ ring located along the perimeter of the facility. Within this facility, a $2 \mathrm{~m}$ diameter methanol pool was placed at ground level (about $3 \mathrm{~m}$ above the basement floor) and ignited. A portion of the grated floor of the facility was covered by a $6 \mathrm{~m}$ diameter steel shroud to prevent spilling into the basement. The pool was recessed so that the top lip was at the same height as the shroud. The liquid level was initially set such that the fuel was within 0.6 centimeter of the top of the lip of the $5.6 \mathrm{~cm}$ deep pan at all points. The pool was modeled as flush with the shroud. During the burn, the pool level receded. The liquid pool level was monitored for fuel evaporation rates with pressure transducers. One meter above the pool, a $30 \mathrm{~cm}$ diameter, $2 \mathrm{~m}$ long horizontal cylinder constructed from $3 \mathrm{~mm}$ thick $(1 / 8 \mathrm{inch})$ stainless steel was located off the centerline by $41 \mathrm{~cm}$ (facility centerline to cylinder centerline). The cylinder was instrumented with heat flux gages and thermocouples to measure the heat transfer to the object. A CARS system was utilized to measure the temperature on the centerline of the facility at $1 \mathrm{~m}$ elevation above the surface of the liquid pool. Two tubes were used as guides for the CARS system and housed some of the optics required for the system. These tubes were about $8 \mathrm{~cm} \mathrm{ID,} 64 \mathrm{~cm}$ long, and were insulated with fiber insulation such that they had an external diameter of about $15 \mathrm{~cm}$. They were located $72 \mathrm{~cm}$ apart, leaving a wide gap for the fire to flow through. The tubes were modeled as solid insulation. More details on the tests are found in the test documentation $[1,3]$.

Some of the measured boundary conditions include: 1) $21 \pm 3^{\circ} \mathrm{C}$ wall temperature, 2) $19 \pm 3^{\circ} \mathrm{C}$ liquid fuel and ambient air temperature, 3) $0.82 \pm 0.01 \mathrm{~atm}$ ambient air pressure, and 4) $1.11 \pm 0.08 \mathrm{~mm} / \mathrm{min}$ fuel regression rate with a corresponding nominal mass flux of $0.015 \mathrm{~kg} / \mathrm{m}^{2} \mathrm{~s}$. Many of the numerical boundary conditions employed for this test are listed in Table 1.

The two conjugate (solid) regions were modeled with the conduction code capability: the 304 stainless steel calorimeter and the optical guide tubes. They were assumed to be at ambient temperature initially. Table 2 
lists the properties assumed for the meshes in these regions. These were taken from manufacturer specifications (tube insulation) or from Holman [11] (steel calorimeter). Exposed surfaces were modeled with an emissivity of 0.9 . This is a typical value as measured for the characterized and painted calorimeter. It may be slightly high for other surfaces. But since for the most part the boundaries are far from the fire, the fire predictions are thought to be otherwise fairly insensitive to the assumed values for emissivity.

A.

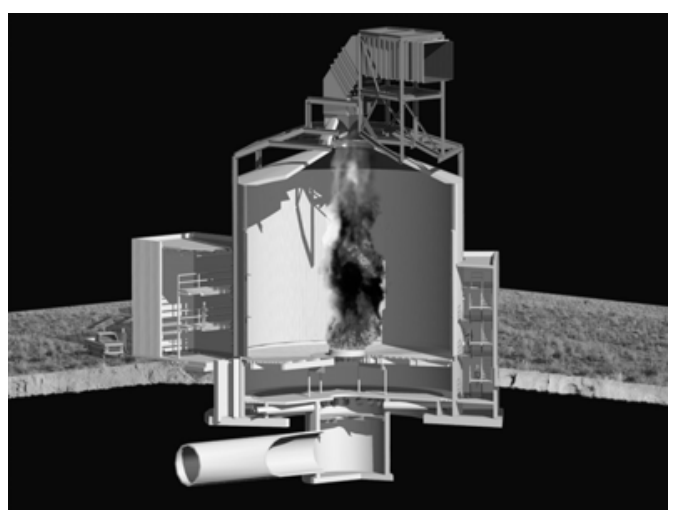

B.

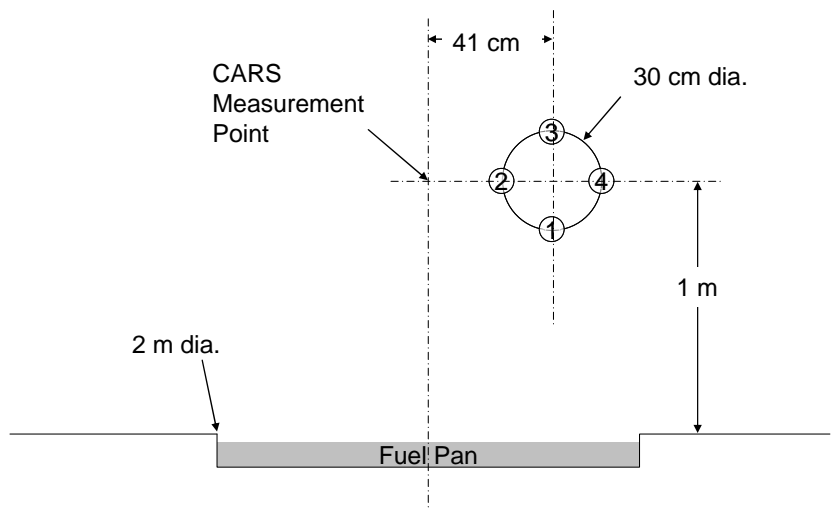

Fig. 1. An illustration (A.) of the flame facility, and (B.) a schematic of the test set-up (not to scale).

Table 1. Principal boundary Conditions used in these calculations.

\begin{tabular}{|c|c|c|c|c|}
\hline Boundary & Ref. & Experimental Value & Fuego boundary & Units \\
\hline $\begin{array}{l}60 \mathrm{~m}^{2} \text { air inlet in the } \\
\text { sub-basement perimeter } \\
\text { ring } 3 \mathrm{~m} \text { below the fire }\end{array}$ & [1] & 58 standard $\mathrm{m}^{3} / \mathrm{s}$ & Air inflow at 0.90 & $\mathrm{~m} / \mathrm{s}$ \\
\hline Basement walls & NA & $\begin{array}{l}\text { Mostly concrete, with un- } \\
\text { measured wall temperatures } \\
\text { unlikely to vary significantly } \\
\text { from ambient }\end{array}$ & Wall at 293 & $\mathrm{~K}$ \\
\hline Water cooled walls & {$[1]$} & $\begin{array}{l}\text { Water cooled: } 21 \pm 3^{\circ} \mathrm{C} \text { from } \\
\text { measurements }\end{array}$ & Wall at 293 & $\mathrm{~K}$ \\
\hline Fuel pan shroud & NA & No measurements & Wall at 293 & $\mathrm{~K}$ \\
\hline Fuel pan & [1] & $0.015 \mathrm{~kg} / \mathrm{m}^{2} \mathrm{~s}$ & $\begin{array}{l}\text { Fuel Inflow at } 0.014 \\
\text { Fuel temperature at } 450\end{array}$ & $\begin{array}{l}\mathrm{m} / \mathrm{s} \\
\mathrm{K}\end{array}$ \\
\hline Grated floor & NA & $\begin{array}{l}\text { Steel grating: examination } \\
\text { suggests high porosity non- } \\
\text { uniform grating }\end{array}$ & $\begin{array}{l}\text { Porous media with gas } \\
\text { volume fraction of } 0.9,0.1 \mathrm{~m} \\
\text { diameter particles mapped } \\
\text { onto the mesh }\end{array}$ & \\
\hline Ceiling & NA & Fiber-insulated metal frame roof & Wall at 293 & $\mathrm{~K}$ \\
\hline Calorimeter & NA & Variable & $\begin{array}{l}\text { Conjugate boundary with the } \\
\text { calorimeter solid model }\end{array}$ & \\
\hline Optical tubes & NA & Variable & $\begin{array}{l}\text { Conjugate boundary with the } \\
\text { tube solid model }\end{array}$ & \\
\hline Outflow & NA & Not measured & $\begin{array}{l}\text { A standard constant pressure } \\
\text { outflow boundary condition }\end{array}$ & \\
\hline
\end{tabular}

Table 2. Assumed conjugate object properties.

\begin{tabular}{|l|c|c|c|}
\hline Object & Specific Heat & Thermal Conductivity & Density \\
\hline Calorimeter & $473 \mathrm{~J} / \mathrm{kgK}$ & $43 \mathrm{~W} / \mathrm{mK}$ & $7801 \mathrm{~kg} / \mathrm{m}^{3}$ \\
\hline Optical tubes & $450 \mathrm{~J} / \mathrm{kgK}$ & $0.0678 \mathrm{~W} / \mathrm{mK}$ & $96.11 \mathrm{~kg} / \mathrm{m}^{3}$ \\
\hline
\end{tabular}

In support of the validation calculations, a convergence study is performed. Convergence can be thought of in many ways. There is the convergence of the numerical equations that can be monitored by the reduction 
in residual values. There is also discretization error, including mesh convergence. Generally, a more refined mesh is expected to give an improved representation of the prediction. Also, there is a time convergence. With smaller time steps, the smaller time scales fluctuations can be resolved in the model. The time and length scale convergence are interrelated, and often managed through the dimensionless Courant Fredrich-Lewis number $(C F L)$. This is defined as $u \Delta t / \Delta x$ where $t$ is time, $x$ is a characteristic length of a cell, and $u$ is the velocity magnitude. Radiation modeling typically involves discretization.

Time accuracy is thought to be adequate when spatial convergence is good and an adequate CFL number (less than 1.0) is retained as was assumed herein. However, time convergence is often demonstrated by rerunning the simulation with a reduced time step and showing that the results are insensitive to the magnitude of the time step used. The calculations presented in the results section were all performed with the mean CFL number below 1.0 as extracted from the maximum across the domain for each time step. Spatial convergence is typically achieved or demonstrated by progressive refinement of the domain and comparing results. The effect of this discretization is examined in detail later in this paper. Radiation discretization has been examined in other studies [12], and found to be relatively insensitive when examining locations engulfed in a fire, and is thus not considered herein. A Picard loop (PL) is a non-linear iteration taken within a time step. The effect of the number of Picard loops taken is examined herein.

Several computational meshes have been formulated for this study, which include four levels of refinement. The meshes employed were developed and tested iteratively based on judgment of the experienced analyst. Table 3 shows details of the meshes employed. All of the fluid meshes are based on uniform refinement of the coarse mesh except for the very coarse mesh. The meshes refined from the coarse then vary in size approximately with integer powers of $3\left(2^{3}: 8\right.$, and $\left.3^{3}: 27\right)$. The very coarse mesh is created by selected non-uniform coarsening. The very coarse mesh was not expected to yield acceptable answers, but was included because it did not require extraordinary resource and would provide additional trend data at minimal cost. More refinement existed near the objects and the pool surface, with a nominal mesh spacing of about $4 \mathrm{~cm}$ (1.5 inches) around the calorimeter for the coarse mesh. The medium mesh had double the resolution (about $2 \mathrm{~cm}$ mesh spacing), and the fine mesh had triple $(1.3 \mathrm{~cm}$ mesh spacing). None of the conjugate (conduction) meshes were refined in the convergence analysis.

Table 3. The meshes for the convergence study.

\begin{tabular}{|l|l|r|l|l|}
\hline \multicolumn{1}{|c|}{ Mesh } & Refinement & \multicolumn{1}{c|}{ Nodes } & Element Type & \multicolumn{1}{c|}{ Calculation } \\
\hline Fluid & Very Coarse & 47,509 & Unstructured Hex & Fluid/Radiation \\
\hline Fluid & Coarse & 258,146 & Unstructured Hex & Fluid/Radiation \\
\hline Fluid & Medium & $2,030,263$ & Unstructured Hex & Fluid/Radiation \\
\hline Fluid & Fine & $6,812,918$ & Unstructured Hex & Fluid/Radiation \\
\hline Calorimeter & Nominal & 94,014 & Unstructured Hex & Conduction \\
\hline Tubes & Nominal & 105,602 & Unstructured Hex & Conduction \\
\hline
\end{tabular}

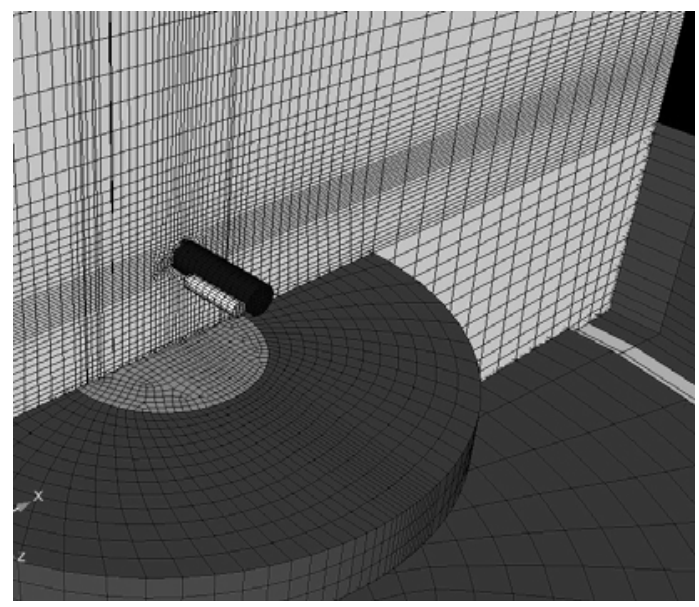

Fig. 2. A plot of the coarse mesh with a cut-plane through the fluid mesh used to illustrate mesh spacing. 
All Fuego calculations reported herein were performed on Sandia National Laboratories' Thunderbird cluster that has 4,480 dual 3.6 GHz Intel EM64T compute nodes operating on Red Hat Enterprise Linux WS release 4.

\section{CONVERGENCE STUDY RESULTS}

Convergence is determined by examining scalar predictions averaged over steady-state portions of the results. After 2 seconds, the fire predictions were generally at a steady-state around the object. Eight seconds of prediction time were averaged at the output rate of the code $(\leq 0.1 \mathrm{~s}$ for all variables). The scalar predictions are then compared for all calculation subsets. Table 4 lists the variables that are examined for determining convergence and explains the abbreviated names used in subsequent plots. Three points of evaluation were used. One is the point where Coherent Anti-stokes Raman Spectroscopy (CARS) temperature measurements were performed in the gas phase. The other points were (1) the bottom center point on the calorimeter, and (2) the point $90^{\circ}$ inward of point (1) on the calorimeter directly across from the CARS point. Corresponding measurements for each variable were taken experimentally.

Table 4. A description of scalar variables plotted for the convergence study.

\begin{tabular}{|l|c|l|}
\hline Abbreviation & Units & Description \\
\hline Tgas & $\mathrm{K}$ & The gas temperature at the CARS measurement point. \\
\hline Tobj1 & $\mathrm{K}$ & Predicted calorimeter temperature at the surface of the calorimeter at point (1) \\
\hline Tobj2 & $\mathrm{K}$ & Predicted calorimeter temperature at the surface of the calorimeter at point (2) \\
\hline Tinf1 & $\mathrm{K}$ & The gas phase temperature in the cell adjacent to the calorimeter at point (1) \\
\hline Tinf2 & $\mathrm{K}$ & The gas phase temperature in the cell adjacent to the calorimeter at point (2) \\
\hline Qconv1 & $\mathrm{W} / \mathrm{m}^{2}$ & The convective heat flux on the surface of the calorimeter at (1) \\
\hline Qconv2 & $\mathrm{W} / \mathrm{m}^{2}$ & The convective heat flux on the surface of the calorimeter at (2) \\
\hline Qrad1 & $\mathrm{W} / \mathrm{m}^{2}$ & The incident radiative heat flux on the surface of the calorimeter at (1) \\
\hline Qrad2 & $\mathrm{W} / \mathrm{m}^{2}$ & The incident radiative heat flux on the surface of the calorimeter at (2) \\
\hline H1 & $\mathrm{W} / \mathrm{m}^{2} \mathrm{~K}$ & The convective heat transfer coefficient on the surface of the calorimeter at (1) \\
\hline H2 & $\mathrm{W} / \mathrm{m}^{2} \mathrm{~K}$ & The convective heat transfer coefficient on the surface of the calorimeter at (2) \\
\hline V_average & $\mathrm{m} / \mathrm{s}$ & The vertical velocity component at the CARS measurement point.
\end{tabular}

Cases were run with 1, 3, 5, and 7 PL for the coarse and very coarse meshes. It was intended to run the medium mesh for 1,3 , and 5 PL. The case with 1 PL was not stable, and no results significantly beyond 1 second could be achieved for this case (even when launching from a restart of a case with 3 or 5 PL). A single fine mesh case was run with $3 \mathrm{PL}$. Figure 3 shows prediction results for the two meshes. In a previous study [12], we found the second non-linear step (i.e., the second Picard loop) induces an increase in the non-linear residual, and requires several more to recover. We generally find simulation predictions with Fuego to be relatively similar with $5 \mathrm{PL}$ and beyond for this type of transient problem. Figure 3a shows that with the exception of the V_average predictions that the $5 \mathrm{PL}$ and $7 \mathrm{PL}$ predictions are in reasonable agreement. From this evidence, we conclude that running with $5 \mathrm{PL}$ is sufficient for this study. The 3 PL results indicate some divergence occurring after the first non-linear iteration.

Figure $3 \mathrm{~b}$ shows the limited prediction results from the medium mesh. Since only two results are present, there is not enough to conclusively demonstrate a trend or convergence. A case with 7 PL was not run due to computational resource limitations at the time this work was performed. The medium mesh trends in Fig. $3 b$ are mostly similar to those found for the same step change with the coarse mesh in Fig.3a.

Mesh convergence is demonstrated as scalars converge for fixed number of Picard loops and different sized meshes. The test matrix was designed to have mesh convergence prediction results for 1,3 , and 5 PL. Since the medium mesh results were not available for $1 \mathrm{PL}$, the existing results are not plotted. Figure $4 \mathrm{a}$ and $4 \mathrm{~b}$ show the prediction results for 3 and 5 PL respectively. 


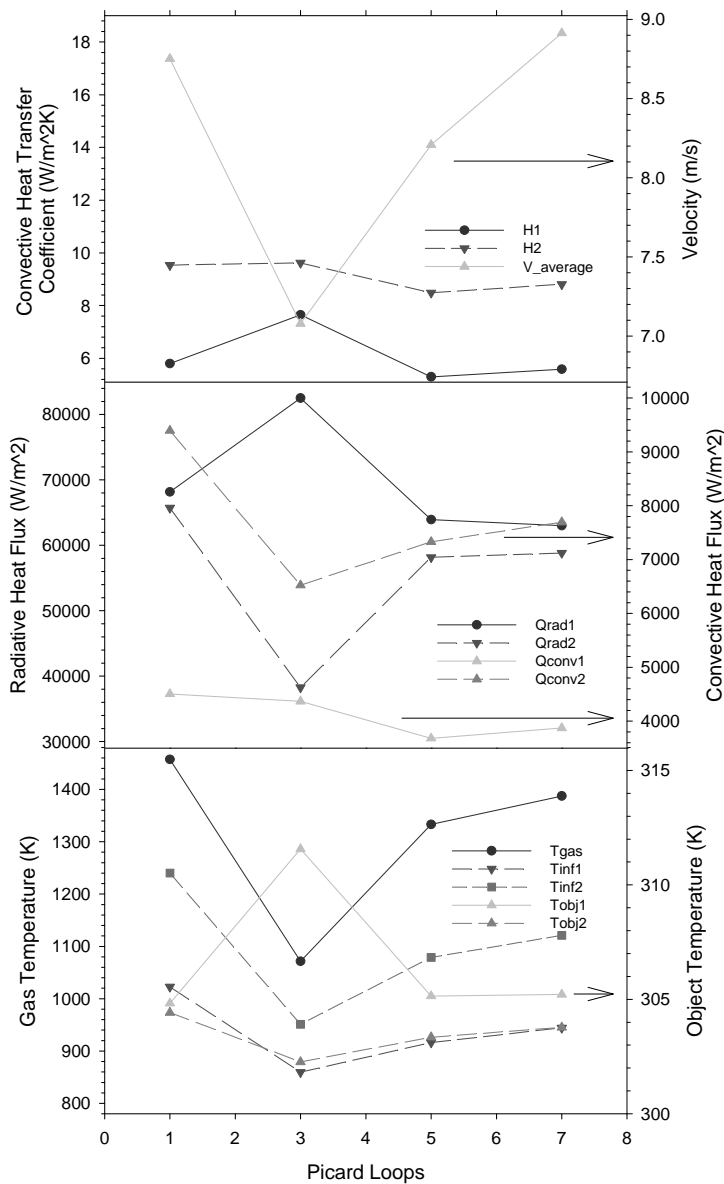

3a) Scalar predictions for the coarse mesh as a function of Picard loops

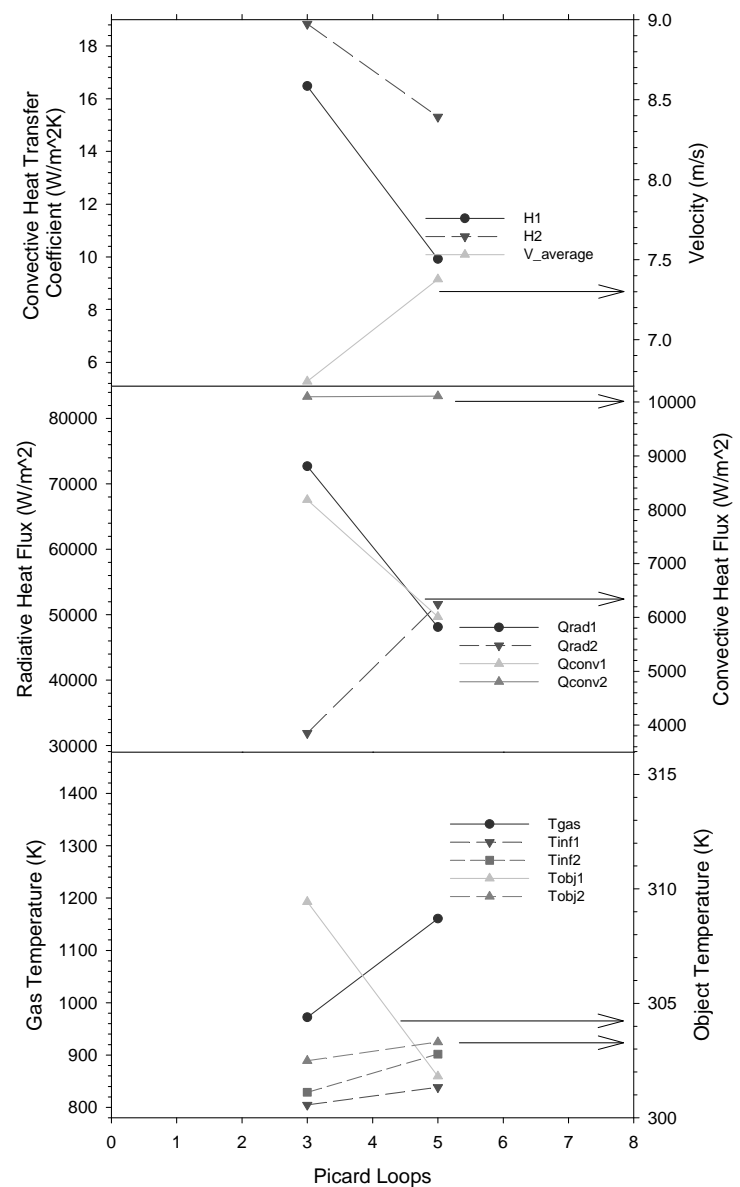

3b) Scalar predictions for the medium mesh as a function of Picard loops

Fig. 3. Scalar predictions for the medium mesh as a function of Picard loops

The Fig.4a results for 3 PL are potentially insufficiently converged (see Fig.3a). The 3 PL results in Fig. 4b are therefore considered secondary to those found with 5 PL (Fig. 4b; because of improved model convergence). The only fine mesh calculation was completed for 3 PL. Since trends are considered important, we have chosen to include these 3 PL results. Temperatures and radiative flux predictions in Fig. 4a suggest some degree of mesh convergence for the radiative heat fluxes and most of the temperatures. A particular concern is found in the temperatures predicted in Fig. 4b. For progressive mesh refinement, as the mesh is increasingly resolved the temperatures trend downward. This is indicative of a need for added mesh refinement, and a significant sensitivity to the mesh. Incident radiation flux predictions also consistently trend downward. There is an interesting trend in the convective heat transfer coefficient. For the very coarse and coarse meshes, the coefficient is in the single digits around $8 \mathrm{~W} / \mathrm{m}^{2} \mathrm{~K}$. There is a significant increase in the convective heat transfer coefficient for the medium mesh to values of 10 and $15 \mathrm{~W} / \mathrm{m}^{2} \mathrm{~K}$. Examining textbook values for free convection from Holman [11], the convective heat transfer coefficient is expected to be in the teens, and is probably too low in the single digits. Examining characteristic length $\mathrm{y}+$ values in the output of the code (coarse with peak $\mathrm{y}+$ around 200, and medium with peak $y^{+}$around 100), it appears that the boundary layer wall functions are inadequately resolved until the resolution achieved with the medium mesh. The convective flux predictions follow the convective heat transfer coefficient predictions despite the temperatures trending downward. Therefore, the medium mesh is thought to be significantly more accurate than the coarse mesh for resolving convection. Results with 3 PL from Fig. 4a are qualitatively quite similar to the 5 PL results in Fig. 4b, and by extrapolation one may assume that the fine mesh with 5 PL might yield similar trends. 


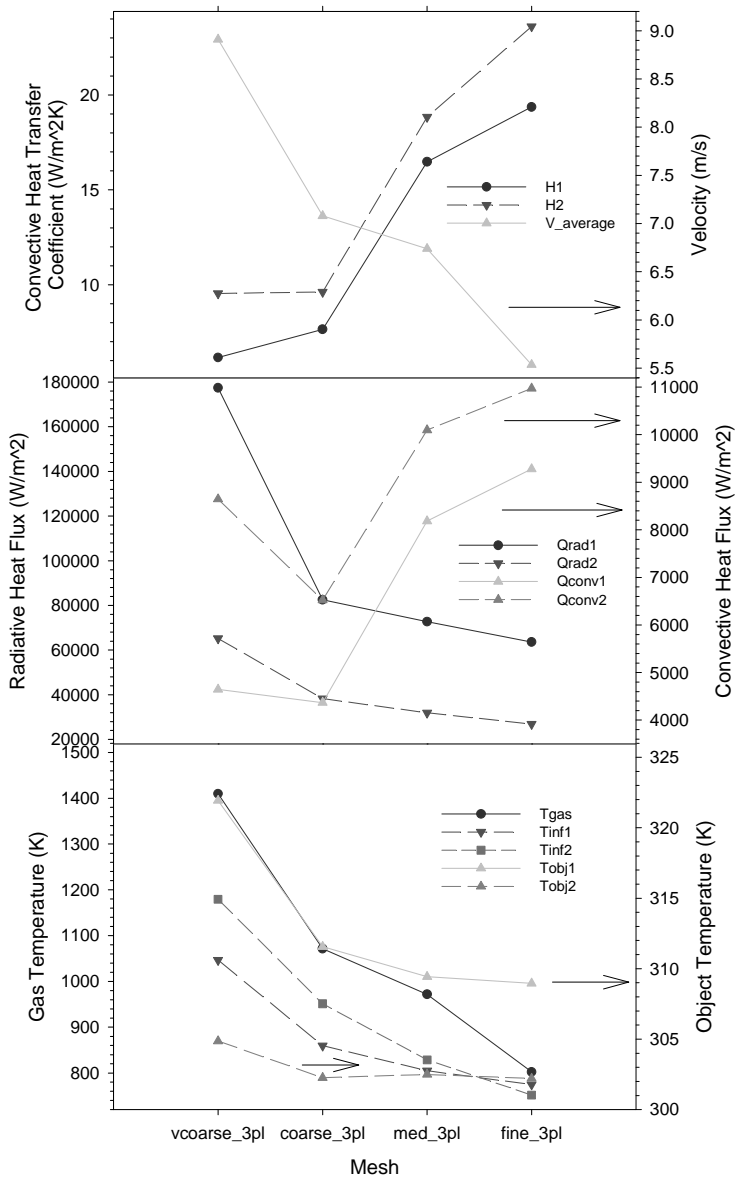

4a) Scalar predictions for $3 \mathrm{PL}$ as a function of mesh resolution

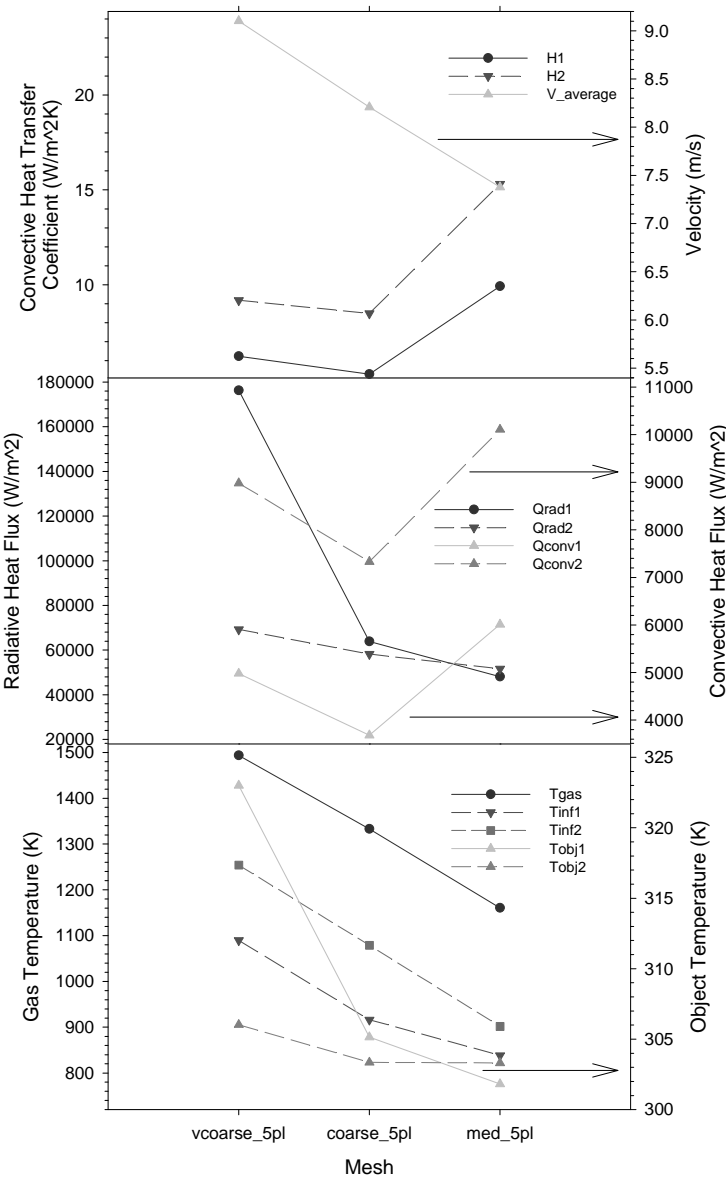

4b) Scalar predictions for $5 \mathrm{PL}$ as a function of mesh resolution

Fig. 4. Scalar predictions for 3 and 5 PL as a function of mesh resolution

Further examination of these results (not presented here) suggested a need for added convergence of the momentum and continuity equations. For the rest of the results, the number of solution iterations for these equations is doubled. This explains the quantitative disparity in the subsequent results with those presented in this section. We expect that the results in the subsequent section would display similar trends to those demonstrated in this section.

\section{VALIDATION COMPARISON RESULTS}

Validation involves quantifying the degree of accuracy of model predictions for an intended application [13-14]. Comparisons are made between the simulation results and the experimental data to quantify the accuracy of Fuego to predict heat flux to an object and the time-resolved gas temperature in a large-scale methanol fire. Estimating the uncertainty in both the experimental data and simulation is an important aspect of validation. As demonstrated in the previous section, the simulation has a dependence on the finite element mesh selected. Using Roache's Grid Convergence Index [15] an uncertainty is estimated for the effect of the mesh density on the simulation. The simulation also depends on the uncertain simulation inputs. The effect of uncertainty in the simulation inputs is propagated through the simulation to provide uncertainty estimates for the simulation outputs. The simulation with its uncertainty is compared to a suite of experiments. The suite of experiments and estimates of the measurement uncertainty are used to define the uncertainty in the experimental data.

Comparisons are shown for the heat flux to the calorimeter at four locations (as illustrated in Fig. 1-B). Heat flux gages that measure the total incident heat flux and radiative heat flux on the calorimeter were 
used in the experiments [1]. From these two heat flux measurements, the convective heat flux can be calculated. Heat flux comparisons are shown for the total, radiative, and convective heat fluxes. Additionally, a comparison is shown for the relative contribution of convection to the total heat flux. The time-resolved temperature at a location in the fire, measured with CARS [1], is also compared.

A suite of nine experiments is available for comparison to the Fuego simulation results for the heat flux [1]. The time-averaged flux is used in the comparison. Each experiment in the suite had nominally the same experimental input conditions. The reported heat fluxes for an experiment are time-averaged over a period in which the measured flux was observed to have a constant mean value. The Fuego simulation was run with the nominally identical boundary conditions (Table 1). Although there is some uncertainty in these boundary conditions, their effect is expected to be insignificant compared to the effect of mesh density and uncertainty in the selected simulation inputs.

\section{Heat Flux Comparisons}

The time-averaged heat fluxes are compared in Fig. 5; comparison of the total heat flux (5a), radiative heat flux (5b), convection heat flux (5c), and the fraction of the total heat flux represented by convection (5d) are shown. Each plot in Fig. 5 compares the time-averaged heat flux from the experiment with the simulation value. The experimental values at each measurement point represent the mean of the heat fluxes measured in the nine experiments with a $95 \%$ probability uncertainty range shown for the combination of the experimental variation in the heat flux and the heat flux measurement error [1]. The simulation values for the coarse and medium meshes, both with 5 Picard loops, are shown in each figure. The uncertainty range shown on the medium mesh simulation value is a $95 \%$ uncertainty interval for the effect of mesh density based on the Grid Convergence Index, which is discussed next.

Uncertainty estimates can be calculated for the effect of the mesh density with the Grid Convergence Index (GCI) [15]. The GCI is based on Richardson extrapolation and uses a factor of safety to convert an error estimate to a $95 \%$ numerical uncertainty estimate. The GCI is defined as:

$G C l=\frac{F_{s} e_{a}^{21}}{r_{21}^{p}-1}$

In Eq. (1), $F_{s}$ is the factor of safety, $p$ is the order of convergence of the simulation output, $e_{a}$ is the relative difference between the simulation outputs on two mesh densities, and $r_{21}$ is the mesh refinement ratio. The relative difference and mesh refinement ratio are defined as:

$$
e_{a}^{21}=\left|\frac{\phi_{1}-\phi_{2}}{\phi_{1}}\right| \quad r_{21}=\frac{h_{2}}{h_{1}}
$$

In Eq. (2), $\phi_{1}$ and $\phi_{2}$ are the simulation outputs on mesh 1 and mesh 2 and $h_{1}$ and $h_{2}$ are characteristic mesh size measures. The magnitude of the factor of safety $\left(F_{s}\right)$, which is a scaling factor to convert the error estimate to an uncertainty estimate, has been empirically studied [15]. Roach recommended a magnitude of $F_{s}=3$ to convert the error estimate to a $95 \%$ uncertainty estimate in two mesh convergence studies. A smaller factor of safety $\left(F_{s}=1.25\right)$ is recommended when three meshes are included in the convergence study (with three meshes the observed $p$ is estimated) and the sequence of meshes is refined in a structured manner. Because only two meshes are used here (1-Medium, 2-Coarse) the observed order of convergence can not be estimated, and $p=1$ is assumed as a conservative estimate with $F_{s}=3$ in Eq. (1). A mesh refinement ratio $\left(r_{21}\right)$ of 2 was used in the mesh convergence study.

The results in Fig. 5 compare the mean (from the nine tests) experimentally measured heat fluxes and their uncertainty with the simulation heat fluxes and their numerical uncertainty estimated from the GCI. The experimental uncertainty is a $95 \%$ probability uncertainty range shown for the combination of the variation in the measured heat flux and the heat flux measurement error [1]. The uncertainty estimate assigned to the medium mesh simulation in Fig. 5 prescribes the range within which the simulation result with no 
discretization error would reside with $95 \%$ probability (given Roache's empirical studies [15]). The simulation values over-predict the measured magnitude of the total (Fig. 5a) and radiative (Fig. 5b) heat fluxes. The convective heat flux is under-predicted at most locations by the simulation. In general, the simulation heat flux values approach the experimental values as the mesh is refined from coarse to medium; better agreement between the measurements and simulations is obtained as the mesh is refined. However, the uncertainty estimates for the effect of mesh discretization are significant, ranging from $15 \%$ to over $100 \%$. At location 4 the numerical uncertainty estimate for the radiative heat flux, Fig. 5b, included negative values. This means the simulation values are sensitive to the mesh at these locations and higher mesh density is needed to resolve the simulation.
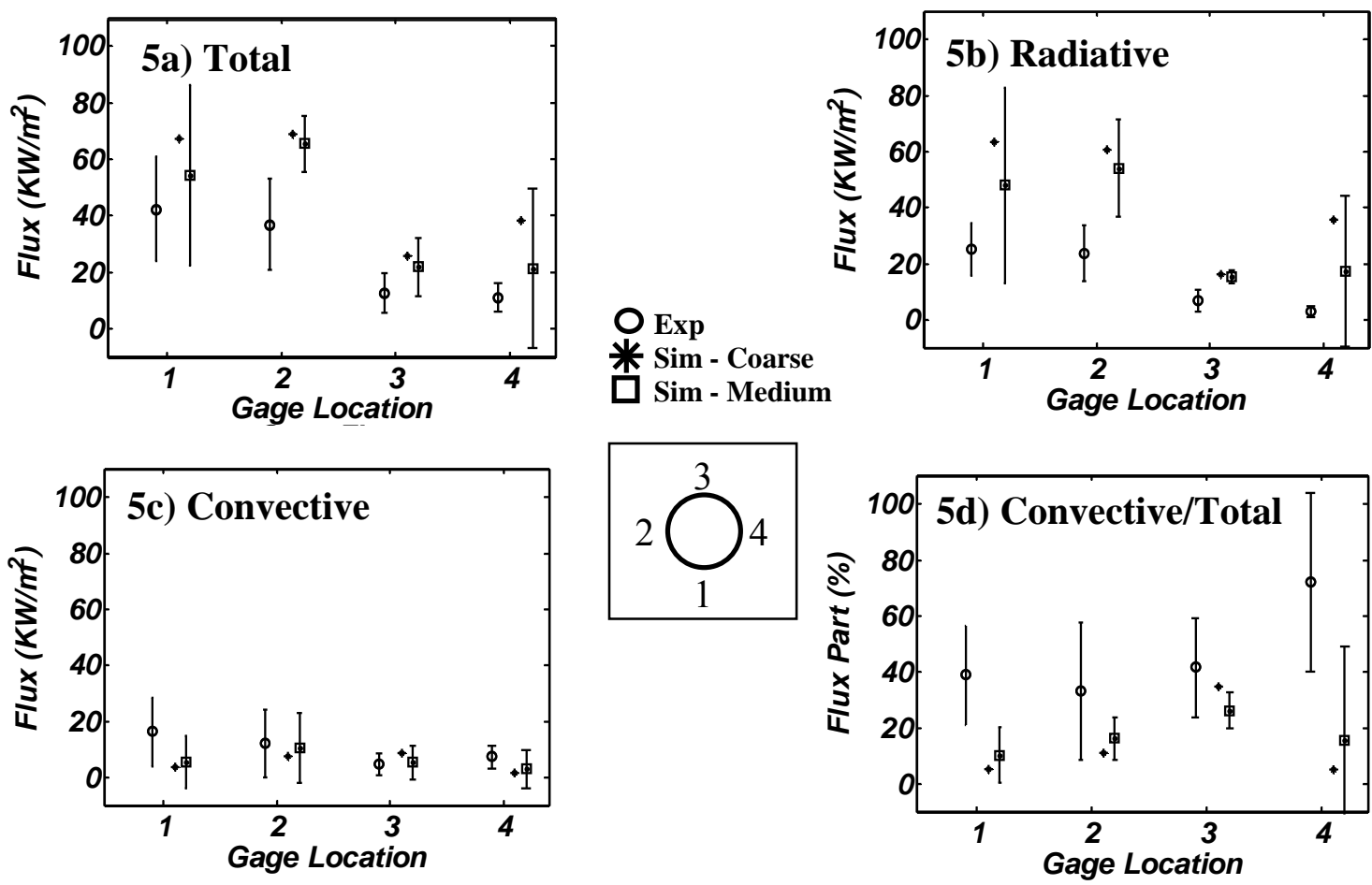

Fig. 5. Time-averaged heat flux comparison of measurements and simulations at the four gage locations.

An uncertainty analysis was also performed to estimate the effect of uncertain input parameters on the simulation output (heat flux). The uncertainty in four simulation input parameters was propagated through the simulation. The input parameters included in the analysis and the uncertainties assigned to the parameters are selected based on analyst judgment; the parameters are listed in Table 5. The uncertainty magnitudes were taken as a standard deviation in the parameter.

Table 5. Uncertain simulation input parameters and the magnitude of the uncertainty.

\begin{tabular}{|l|l|}
\hline Input Parameter $\left(p_{i}\right)$ & Uncertainty $\left(\sigma_{p_{i}}, \%\right)$ \\
\hline Convection Coefficient (Conv) & 50 \\
\hline Flame Volume Coefficient (FVC) & 15 \\
\hline Heat of Combustion (HOC) & 2.5 \\
\hline Flame Loading Coefficient (FLC) & 15 \\
\hline
\end{tabular}

A mean-value approach was used to propagate input parameter uncertainty through the simulation [16]. The propagation equation for a mean-value approach is:

$\sigma_{r}=\left[\sum_{i=1}^{N_{p}}\left(\frac{\partial r}{\partial p_{i}} \sigma_{p_{i}}\right)^{2}\right]^{1 / 2}$ 
The uncertainty (standard deviation) in the simulation response, $\sigma_{r}$, (i.e., total, radiative, and convective heat fluxes) is estimated from the partial derivatives of the simulation response with respect to each parameter, $\partial r / \partial p_{i}$, and the uncertainty (standard deviation), $\sigma_{p i}$, in each parameter. The approach requires running the simulation while separately perturbing each parameter to approximate the partial derivatives. A forward difference numerical approximation was used to estimate the partial derivatives. The magnitude of the perturbation was related to the standard deviation (uncertainty) of the parameter. Because a forward difference approximation was used to approximate the partial derivatives, the linearity of the simulation in the parameter space could not be assessed. It is recognized that the linearity assumption is an approximation that may not be satisfied by the complex simulation. However, a linear analysis is insightful to understanding the impact of simulation input uncertainties and feasible given the computational expense of performing the simulations. Due to computational limitations, uncertainty in the simulation inputs could only be assessed for the simulation with the coarse mesh. Experience has demonstrated that the partial derivatives of the simulation (and simulation uncertainty) also have a dependence on the mesh density.

Uncertainty estimates for the effect of mesh density $(\mathrm{GCI})$ and simulation input uncertainty $\left(\sigma_{r}\right)$ were developed above. These simulation uncertainty estimates for the heat fluxes are listed in Table 6 . The percent uncertainty in the simulated values is tabulated for the various simulation outputs considered and the four gage locations. The GCI is an uncertainty range tied to a $95 \%$ probability. The uncertainty estimate for input parameter uncertainty, $\sigma_{r}$, is multiplied by two (assuming a normal distribution) and normalized to give a percent uncertainty tied to a $95 \%$ probability.

The uncertainty estimates for mesh density and due to input parameter uncertainty have generally comparable magnitudes. Some estimates of both uncertainties are near, or exceed, 100 percent; uncertainty in the convective flux is an example. Reasons for this are elucidated in the convergence results section. For the GCI this means there is a large relative difference in the simulation values on the two meshes, and mesh refinement is significant to the comparison with data. For input uncertainty, this means the simulation is sensitive to an input parameter with significant uncertainty, and the comparison with experimental data depends on the value selected for that input parameter.

In all instances except gage 3 for the convective/total, the medium mesh results are closer to the nominal data point than the coarse mesh results. Whereas the medium mesh results in Fig. 5 generally showed overlap with the data, the coarse predictions with modeling uncertainty estimates do not. In most cases, the observed discrepancy between the measurement and simulation is within the combined uncertainty of the simulation and experiment; the exception is predicting the convective/total fraction. The uncertainty needs to be reduced (with higher mesh resolution and/or an improved understanding of the inputs) to identify simulation bias.

Table 6. Uncertainty in the simulation values at the four gage locations.

\begin{tabular}{|l|l|l|l|l|l|l|l|l|}
\hline Gage & \multicolumn{2}{|c|}{ Total Heat Flux } & \multicolumn{2}{|l|}{ Radiative Heat Flux } & \multicolumn{2}{|l|}{ Convective Heat Flux } & \multicolumn{2}{l|}{ Convective/Total } \\
\hline & $\begin{array}{l}\text { GCI } \\
(\%)\end{array}$ & $\begin{array}{l}2 \sigma_{Q_{\text {tot }}} / Q_{\text {tot }} \\
(\%)\end{array}$ & $\begin{array}{l}\text { GCI } \\
(\%)\end{array}$ & $\begin{array}{l}2 \sigma_{Q_{\text {rad }}} / Q_{\text {rad }} \\
(\%)\end{array}$ & $\begin{array}{l}\text { GCI } \\
(\%)\end{array}$ & $\begin{array}{l}2 \sigma_{Q_{\text {conv }}} / Q_{\text {conv }} \\
(\%)\end{array}$ & $\begin{array}{l}\text { GCI } \\
(\%)\end{array}$ & $2 \frac{\sigma_{Q_{\text {conv }}} / Q_{\text {tot }}}{\left(Q_{\text {conv }} / Q_{\text {tot }}\right)}$ \\
\hline 1 & 59.3 & 49.7 & 72.3 & 51.4 & 165 & 113 & 93.0 & 112 \\
\hline 2 & 15.3 & 18.8 & 32.0 & 18.8 & 115 & 91.2 & 45.6 & 84.0 \\
\hline 3 & 48.1 & 29.7 & 15.0 & 21.0 & 109 & 94.9 & 24.2 & 68.9 \\
\hline 4 & 136 & 26.0 & 155 & 27.8 & 204 & 135 & 208 & 136 \\
\hline
\end{tabular}

\section{Gas Temperature Comparison}

Time-resolved gas temperature is also measured in the experiment. The Fuego EDC predicted temperatures are cell mean temperatures, with a presumed distribution according to the progress variable. The progress variable is not used to further broaden predicted distributions in this section. The histogram of the measured gas temperature is shown in Fig. 6a at the CARS measurement location given in Fig. 1. The measured gas temperature is relatively uniformly distributed over the range from $300 \mathrm{~K}$ to $1900 \mathrm{~K}$. The histogram of the simulation at this location is shown in Fig. $6 \mathrm{~b}$ and $6 \mathrm{c}$ for the coarse and medium mesh, 
respectively. The simulation with the coarse mesh has a definite peak near $1500 \mathrm{~K}$ but has a less defined peak for the medium mesh. Also, the range of the simulation is wider for the medium mesh than for the coarse mesh. The medium mesh is more uniformly distributed than the coarse mesh. In these regards, the medium mesh results better agree with the experimental data.

6a) Experiment (CARS)

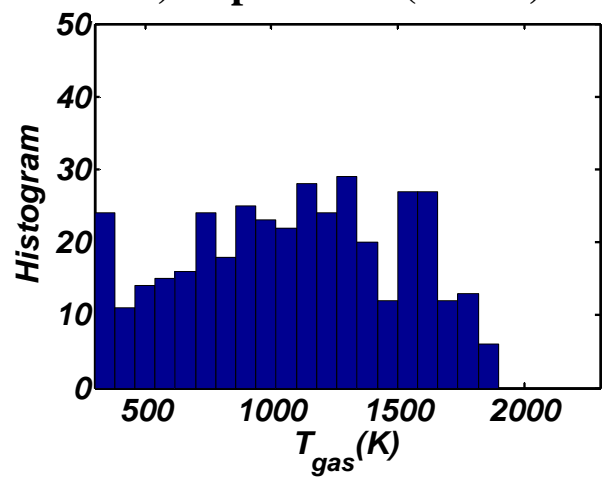

6c) Sim - Medium

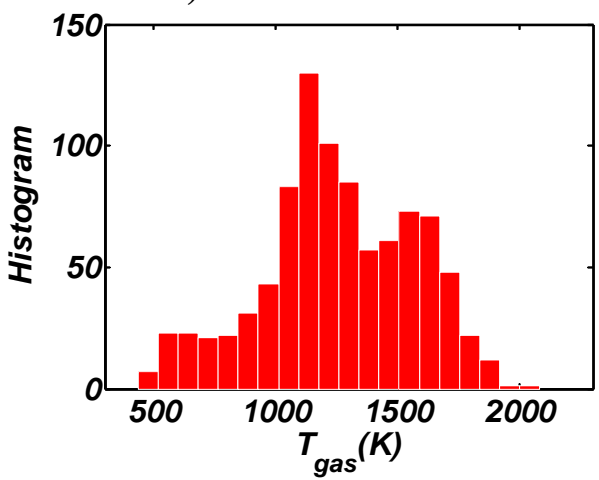

6b) Sim -Coarse

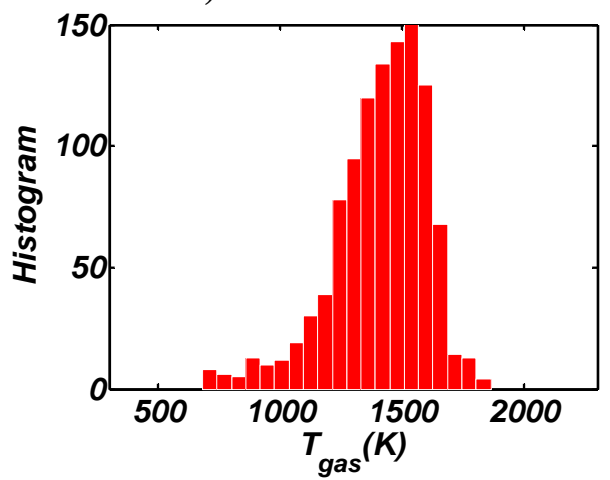

6d) CDF Comparison

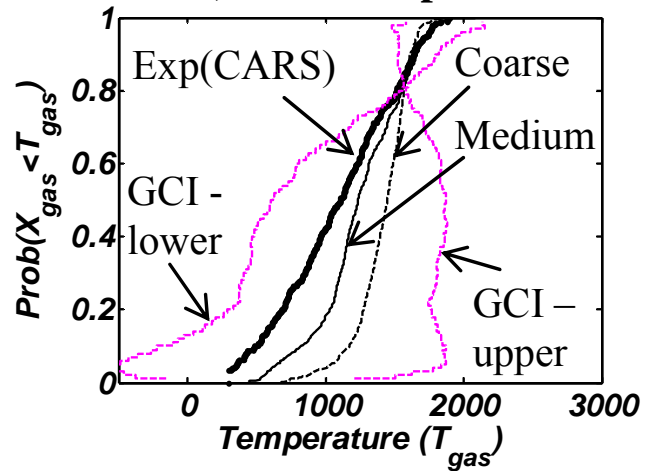

Fig. 6. Comparison of measurements and simulations of the time-resolved temperature.

The gas temperature demonstrates random variation. One measure of random variation is the cumulative distribution function. The cumulative distribution function (CDF) gives the probability that a random variable, $X_{\text {gas }}$, takes on a value less than or equal to measured/simulated gas temperature $\left(T_{\text {gas }}\right)$. The empirical cumulative distribution functions are estimated from the measured and simulation gas temperature histograms. A comparison between the measured and simulated CDFs is shown in Fig. 6d with GCI estimated uncertainty bounds ( $95 \%$ confidence). The medium mesh results are closer to the data, and the data fall well within the bounds of simulation uncertainties due to the GCI. A simulation with a more refined mesh is needed to further resolve the comparison with the experimental data.

\section{CONCLUSIONS}

New high-fidelity data have yielded excellent context for performing validation of fire CFD models. Validation comparisons for the Fuego code are demonstrated. The convergence study suggests the need for fairly high mesh resolution to properly resolve this problem. Spatial resolution (mesh) uncertainty magnitudes have been estimated. Parametric uncertainties in the models are also estimated. These are shown to be of similar magnitude for the heat flux data. This suggests that increased mesh resolution is a productive pathway to consider for improving simulation uncertainties for this class of problems. Gas temperature histograms are also compared. As the mesh is refined, the distributions appear more similar to the data, further suggesting the benefit of increased resolution.

\section{ACKNOWLEDGEMENTS}

Sandia is a multiprogram laboratory operated by Sandia Corporation, a Lockheed Martin Company, for the United States Department of Energy's National Nuclear Security Administration under contract DE-AC04- 
94AL85000. Funding was provided under the Advanced Scientific Computing (ASC) project. Sean Kearney (SNL) provided helpful discussions and input on the nature of the experimental system.

\section{REFERENCES}

[1] Blanchat, T.K., O’Hern, T., Kearney, S., Ricks, A., and Jernigan, D., "Validation Experiments to Determine Radiation Partitioning of Heat Flux to an Object in a Fully Turbulent Fire," Accepted to the 32nd Symposium (International) on Combustion, Montreal, Canada, August 3-8, 2008.

[2] Sean P. Kearney, Kraig Frederickson, and Thomas W. Grasser, "Dual-pump coherent anti-stokes Raman scattering thermometry in a sooting turbulent pool fire," Accepted to the 32nd Symposium (International) on Combustion, Montreal, Canada, August 3-8, 2008.

[3] Ricks, A., Blanchat, B., Brown, A.L., Dowding, K., and Nicolette, V., "An Experimental and Model Validation Study on the Convection/Radiation Partitioning of Heat Flux to an Object in a Fully Turbulent Fire," SAND report in progress 2008.

[4] Domino, S.P., Moen, C.D., Burns, S.P., and Evans, G.H., "SIERRA/Fuego: A Multi-Mechanics Fire Environment Simulation Tool," AIAA Paper 2003-0149, 41st AIAA Aerospace Sciences Meeting, Reno, NV, January 2003.

[5] Domino, G. Wagner, A. Luketa-Hanlin, A. Black and J. Sutherland "Verification for multimechanics applications," AIAA Paper 2007-1933, 48th AIAA/ASME/ASCE/AHS/ASC Structures, Structural Dynamics, and Materials Conference, Honolulu, HI, 2007.

[6] Tieszen, S.R., Domino, S.P., and Black, A.R., "Validation of a Simple Turbulence Model Suitable for Closure of Temporally-Filtered Navier-Stokes Equations Using a Helium Plume," Sandia National Laboratories report, SAND2005-3210, 58 pages.

[7] Girimaji, S.S., "Partially-Averaged Navier-Stokes Model for Turbulence: A Reynolds-Averaged Navier-Stokes to Direct Numerical Simulation Bridging Method," Journal of Applied Mechanics, May 2006, 73:3, 413-421. http://dx.doi.org/10.1115/1.2151207

[8] Magnussen, B.F., and Hjertager, B.H., "On Mathematical Modeling of Turbulent Combustion with Special Emphasis on Soot Formation and Combustion," 16th Symposium (International) on Combustion, The Combustion Institute, 1977, pp. 719-729.

[9] Magnussen, B.F., "On the Structure of Turbulence and a Generalized Eddy Dissipation Concept for Chemical Reactions in Turbulent Flow," 9th AIAA Sc. Meeting, St. Louis, 1981.

[10] Leckner, B., "Spectral and Total Emissivity of Water Vapor and Carbon Dioxide," Combustion and Flame, vol. 19, 1972, pp. 33 - 48. http://dx.doi.org/10.1016/S0010-2180(72)80084-1

[11] Holman, J.P., Heat Transfer, Seventh Edition, McGraw Hill, Inc, 1990.

[12] Brown, A.L., Evans, G., Gill, W., and Jarboe, D. "Validation Predictions of a $13 \mathrm{~m} / \mathrm{s}$ Cross-wind Fire for Fuego and the University of Waterloo Dataset," SAND2008-0919, 154 pages.

[13] AIAA, Guide for the Verification and Validation of Computational Fluid Dynamics Simulations, AIAA G-077-1998.

[14] ASME, Guide for the Verification and Validation in Computational Solid Mechanics, ASME V\&V10-2006.

[15] Roache, P. J., Verification and Validation in Computational Science and Engineering, Hermosa Publishers, Albuquerque, August 1998.

[16] Coleman, H. W. and Steel, W. G., Experimentation and Uncertainty Analysis for Engineers," Second Edition, Wiley Publishers, New York, 1999. 